ÉGYPTE monde arabe

\section{Égypte/Monde arabe}

15-16 | 1993

Les crises soudanaises des années 80

\title{
L'islam chez les Berti
}

Abdulahi Osman Al-Tom

\section{(2) OpenEdition}

\section{Journals}

Édition électronique

URL : https://journals.openedition.org/ema/1103

DOI : 10.4000/ema.1103

ISSN : 2090-7273

\section{Éditeur}

CEDEJ - Centre d'études et de documentation économiques juridiques et sociales

\section{Édition imprimée}

Date de publication : 31 décembre 1993

Pagination : 249-257

ISSN : 1110-5097

\section{Référence électronique}

Abdulahi Osman Al-Tom, «L'islam chez les Berti », Égypte/Monde arabe [En ligne], 15-16 | 1993, mis en ligne le 08 juillet 2008, consulté le 07 juillet 2022. URL : http://journals.openedition.org/ema/1103 ; DOl : https://doi.org/10.4000/ema. 1103

Ce document a été généré automatiquement le 7 juillet 2022.

Tous droits réservés 


\title{
L'islam chez les Berti
}

\author{
Abdulahi Osman Al-Tom
}

\section{NOTE DE L'ÉDITEUR}

Traduit de l'anglais par Mona Niazi.

1 Les Berti, dont le nombre s'élève à environ 60.000, vivent dans la province du NordDarfour en République du Soudan ${ }^{1}$. Leur économie est une économie de subsistance reposant sur les cultures pluviales et l'élevage du bétail. Leur aliment de base est le millet, leurs principales cultures de rapport les arachides, le sésame, les pastèques et la gomme arabique. Ils élèvent des chèvres, des moutons, des bœufs et des ânes, soit pour le commerce, soit pour leur consommation. Les Béni sont patrilinéaires et vivent selon le mode patrilocal et sédentaire dans de petits villages d'une vingtaine de foyers.

2 La famine de 1983-85 a eu des effets désastreux sur leur économie, anéantissant plus de $75 \%$ du bétail tandis que la majorité de la population ne survivait que grâce aux organismes d'aide. Le système politique des Berti a été de tous temps un système centralisé qui, dans le passé, relevait du sultanat du Darfour. Les Berti sont musulmans et de rite sunnite malikite. Depuis plusieurs générations, ils parlent une variété d'arabe dialectal qui leur est propre.

3 L'adhésion des Berti à l'islam s'est effectuée graduellement au long de plusieurs siècles. On ignore la date de leur première rencontre avec les musulmans. Selon les chroniqueurs, c'est au XIV ${ }^{e}$ siècle que le sultanat du Darfour a été menacé par une incursion arabe, comme l'indique une plainte officielle du sultan présentée au pouvoir musulman égyptien de l'époque (O'Fahey, 1980, 5) et dénonçant en fait des infiltrations individuelles. La position géographique du Darfour et de son sultanat sur la route menant de l'Afrique de l'Ouest vers la Mecque avait conduit plusieurs pèlerins à s'établir au Darfour, soit à l'aller, en direction du Hijaz, soit durant le voyage de retour. On estime à environ un tiers de la population de cette province le nombre de pèlerins d'Afrique de l'Ouest qui s'y sont établis (ibid., 4). 
4 L'adoption de l'islam par la dynastie régnante n'a pas peu contribué à le consolider. Cette dernière entendait ainsi s'assurer la légitimité, ce qui indique que cette religion était déjà largement répandue dans la population du sultanat avant d'être reconnue par le palais. L'islamisation de l'autorité au pouvoir est illustrée par la légende du «sage étranger» qui avait épousé une des jeunes filles de la famille régnante: "La domination des Keira sur les Tunjur est attribuée à Ahmad al-Maqur (Ahmad-auxjambes-coupées), un Arabe d'Afrique du Nord. Les jambes d'Ahmad avaient été coupées par son frère à la suite d'une querelle concernant l'épouse de ce dernier, mais il avait été sauvé du désert et conduit au roi des Fur, Show Dorshid, de la dynastie des Tunjur, monarque mystérieux et tyrannique redouté par ses sujets. Ahmad gagna sa faveur et celle de sa fille en introduisant dans les mœurs de la cour un comportement plus civilisé, notamment de bonnes manières de table. » (ibid., 123)

5 Mais la tribu des Fur n'est pas la seule à avoir fait l'objet d'une telle légende. On la trouve aussi chez les Berti et chez bon nombre d'autres tribus, dont celles des montagnes Nuba (Trimingham 1949,135). Le «Sage» des Berti est Muhammad Yanbar de la lignée des Basangas, lignée à laquelle ont appartenu les rois berti jusqu'à l'abolition du royaume vers la fin des années 60 . Selon la légende, Yanbar aurait été envoyé en mission spéciale, depuis la Mecque, pour renverser le tyran géant Namudu et rétablir la Justice dans la province des Berti. En se dirigeant vers leur territoire, Yanbar puisait sa force dans la méditation. C'est d'ailleurs à celte fin qu'il s'était retiré dans le désert sept années durant. Yanbar ne tarda pas à gagner la confiance du géant grâce à ses qualités d'homme plein de sagesse et à son sens de l'initiative. Cela se révéla dans les changements qu'il introduisit dans le domaine des manières de table et du mariage. Yanbar ouvrit une école coranique et continua de prêcher l'islam. Mais il ne s'en tint pas là : son but ultime était de chasser le tyran et d'instaurer la justice, ce qu'il ne put réaliser que grâce à sa force spirituelle comme cela avait été le cas à son arrivée chez les Berti. Le tyran ne tarda pas à être renversé et chassé et repartit probablement vers la destination mystérieuse d'où il était initialement venu.

6 Cette légende berti présente plusieurs similitudes avec celle des Fur. Malgré les différences de nom et de contexte, la structure du récit mythique est la même. Dans les deux cas, un étranger sage et équitable remplace un tyran autochtone barbare et inique. Cet étranger est originaire de la Mecque, à l'est, qui est pour les Berti une direction de bon augure (voir Holy 1974). L'étranger adhère à la religion «la plus appropriée » dans laquelle il puise sa force, alors que son hôte est un païen qui règne par la répression sur son peuple. Enfin, l'étranger est d'une lignée connue tandis que l'origine de son hôte est obscure et mystérieuse.

7 L'intégration de Yanbar dans la dynastie régnante et son accession au pouvoir permit aux Berti d'adopter une nouvelle idéologie et une nouvelle généalogie. L'islamisation des Berti alla ainsi de pair avec leur arabisation. Ce phénomène est assez répandu dans d'autres parties du nord du Soudan, où plusieurs tribus affirment être affiliées aux Arabes, malgré le nombre limité d'immigrants arabes dans le pays. Chez les Berti, l'origine arabe pourrait être réelle, compte tenu des 99 lignées qui - prétendent-ils existent chez eux. Mais bien que plusieurs Berti prétendent être en possession de copies de leur arbre généalogique, je n'ai personnellement trouvé que trois arbres généalogiques appartenant à deux lignées différentes. Yanbar se situe entre les 11e et 13e générations antérieures et les arbres généalogiques remontent au Prophète Muhammad, 34 à 37 générations avant la génération actuelle. La corrélation entre 
l'arabisation et l'islamisation peut être constatée à divers niveaux. Les Berti évoquent d'ailleurs ces deux faits comme s'ils étaient synonymes, ce que prouve par exemple leur conception de la circoncision, considérée par eux comme une pratique musulmane (Lewis, 1966, 52, 68). Pour désigner cette pratique, ils utilisent en effet le terme ya'rib («arabiser», et non 'circoncire') ou disent encore yidakhilhum fi-l-sunna («les taire entrer dans la Sunna »).

Mais l'adhésion des Berti à la Sunna ne signifiait ni une acceptation totale de tous les enseignements de la nouvelle idéologie, ni un renoncement absolu à leurs croyances païennes en faveur de leur nouvelle foi monothéiste. On trouve chez les Berti des croyances et des pratiques d'origine aussi bien islamique que non islamique et, d'une manière générale, leur adhésion à l'islam constitue un phénomène plus collectif qu'individuel. Les rites islamiques sont également célébrés avec beaucoup plus d'enthousiasme lorsqu'il s'agit de pratiques collectives : prières pour obtenir la pluie, rituels de mariages ou d'obsèques... Mais au niveau individuel, les rites islamiques sont complètement négligés. Ainsi par exemple, la prière du matin n'est observée que par quelques rares villageois, en général des vieillards, alors que les prières des fêtes religieuses sont pratiquées par tous les adultes. Pourtant les prières individuelles sont imposées par le Coran et constituent un farad - c'est-à-dire un rite obligatoire dans l'islam -, alors que les prières collectives susmentionnées ne sont qu'une des pratiques inspirées de la vie du Prophète (Sunna) et, partant, ne sont pas obligatoires.

9 L'adhésion des Berti à l'islam a parfois entraîné la réinterprétation de certains concepts religieux. Ainsi, la majorité des villageois berti fabriquent une sorte de bière de millet dont ils consomment d'assez grandes quantités. La plupart sont prêts à se lancer dans une argumentation très élaborée pour prouver que la consommation de cette bière n'est pas prohibée par l'islam. Certains prétendent encore que l'alcool interdit par le Coran est l'alcool distillé et ne concerne pas la bière locale. D'autres affirment que consommer de la bière n'est pas répréhensible puisque sa production et son acquisition ne sont pas interdites par la loi. D'autres enfin considèrent que la consommation de bière est permise tant que celui qui la boit ne cause pas de problèmes à autrui, et comme cette boisson est peu alcoolisée, on considère qu'elle est rarement génératrice d'ivresse.

10 La réinterprétation du dogme islamique par les Berti concerne également leur compréhension des textes coraniques. Leur maîtrise imparfaite de l'arabe coranique les a conduit à imposer leur propre lecture des textes sacrés, lecture qui s'écarte souvent de l'interprétation théologique consacrée. Cela leur a permis de servir leurs intérêts et de rendre certains passages plus adaptés à leur vie quotidienne.

11 Les Berti ont, dans une certaine mesure, adopté le calendrier lunaire islamique. Néanmoins, le système qu'ils ont adopté pour calculer les dates est cyclique, étant donné qu'ils ne connaissent que les mois et non l'année islamique. Le jeûne de Ramadan est rigoureusement observé, surtout par les hommes: son non respect risquerait d'attirer le malheur sur toute la communauté. Le jeûne revêt chez eux une dimension collective. Le fait d'attribuer une dimension collective à une action individuelle peut aussi être constaté en ce qui concerne l'homicide, dont les suites sont normalement réglées par le paiement d'une compensation collective ou "diya " (à propos de la diya chez les Berti, voir Holy, 1974). La diya représente une manière collective d'assumer la faute qui, selon l'islam, retombe sur son auteur. Le versement de la zakât (aumône légale) et autres aumônes est plus ou moins observé par les Berti. 
Le fait de ne pas s'en acquitter entraîne selon eux une menace pour le patrimoine et implique un châtiment inévitable dans l'au-delà. S'abstenir de donner aux pauvres est considéré comme un péché.

12 Autre «pilier » de l'islam : le pèlerinage à la Mecque pour ceux qui en ont les moyens. Comparés aux musulmans d'Afrique de l'Ouest, les Berti sont moins enthousiastes en ce qui concerne le pèlerinage. Contrairement aux Berti, les musulmans d'Afrique de l'Ouest n'acceptent pas que l'on prétexte, pour ne pas effectuer le pèlerinage, de la distance qui sépare leur pays de la Mecque. Même si le pèlerinage confère à celui qui l'accomplit un titre (celui de hajj) et un statut, cela ne représente pas pour les Berti une motivation aussi puissante que pour les musulmans d'Afrique de l'Ouest. Le pèlerinage à la Mecque est souvent remplacé chez les premiers par une visite au tombeau d'un saint local; suivant la coutume répandue dans le nord du Soudan et dans d'autres régions musulmanes. Comme il n'existe pas de mausolée célèbre dans la région des Berti, ces derniers se déplacent jusqu'à ceux d'autres régions. Les quelques tombeaux locaux, peu importants et qui, pour la plupart, appartiennent à des saints non identifiés, reçoivent aussi certains visiteurs.

13 L'idée d'une différenciation sexuelle dans la pratique religieuse, répandue en Afrique Noire et selon laquelle les nommes seraient plus musulmans et les femmes plus «païennes », ou plutôt animistes, existe également chez les Berti. Bien que les femmes participent - quoiqu'en nombre inférieur, de loin, à celui des hommes - aux prières accomplies durant les deux grandes fêtes annuelles, elles ne participent pas aux prières pour la pluie ni à celles que l'on récite lors des catastrophes, de même qu'elles ne prennent pas part aux sacrifices, aux enterrements et aux prières de mariage. Quant à la prière individuelle quotidienne, elles l'accomplissent encore moins que les hommes. En principe, les filles sont admises dans les écoles coraniques, mais leurs effectifs y sont relativement faibles et se limitent aux fillettes de moins de dix ans. Les femmes se réservent surtout les rites animistes qui, parallèlement à l'islam, continuent de prévaloir dans cette région. Ces rites, principalement coutumes ('ada) ou superstitions (sanam), se pratiquent à diverses étapes de la culture du millet et à l'occasion de certaines "crises de l'existence ». Dans son étude sur les Somaliens, Lewis considère que les femmes sont attirées par ce genre de pratiques (par exemple le zâr) parce qu'exclues des autres rites publics (Lewis, 1966, 64). Il existe aussi chez les Berti une dualité intéressante concernant la divination et le traitement des malades. La géomancie islamique (interprétation de signes sur le sable) subsiste parallèlement à d'autres méthodes telles que la divination par les coquillages, par exemple. Le traitement des malades selon les coutumes islamiques classiques est pratiqué par les hommes de religion (fugaha) et constitue une alternative à l'usage des plantes médicinales chez les villageois analphabètes.

14 La réclusion des femmes dans les appartements privés de la maison, assez commune dans l'islam, est moins répandue chez les Berti que dans d'autres sociétés islamiques. Mais l'islam a marqué de son empreinte l'architecture des maisons qui comprennent toujours une section séparée de celle où ont lieu les activités sociales. Les femmes Berti ne doivent toutefois pas être considérées comme des recluses, à l'instar du groupe de femmes libanaises dont Gilsenan a décrit le comportement dans son étude (Gilsenan, 1982). Les femmes Berti se joignent aux hommes dans les champs, sur les marchés, etc. Les grossesses avant le mariage sont fréquentes et ne rencontrent pas la désapprobation sociale comme chez les bédouins arabes. Si les enfants illégitimes sont 
évidemment à éviter, l'illégitimité de l'enfant est vite oubliée. Enfin, contrairement au code ou à la pratique islamique, il suffit de payer une amende ou de prêter serment de ne plus commettre l'adultère pour qu'une telle faute soit considérée comme expiée.

Les Berti établissent une distinction entre politique et religion. Dans leur langue, il n'existe aucun terme pouvant se traduire littéralement par "politique " mais seulement des termes appartenant au champ sémantique du concept qui a pour nom "politique», notamment celui qui s'en approche le plus, hukm (pouvoir). Les Berti expliquent leur volonté de séparer la religion de la politique par des arguments empruntés à leur tradition orale et par leur ascendance. Selon eux, l'arrivée de Yanbar, l'ancêtre de la lignée Basanga, fut suivie peu après par celle de son cousin Muhammad Dawazen (ou Dawazed) qui appartenait à la lignée connue des Charafa. Au terme de l'âpre lutte pour le pouvoir qui opposa longtemps les Basanga aux Charafa, un accord fut conclu en vertu duquel les membres de la lignée des Basanga s'engageaient à limiter leur autorité au domaine politique et à céder aux Charafa l'autorité religieuse. D'après la seule copie de l'arbre généalogique des Charafa que j'ai pu examiner, Dawazen ne se situe que huit générations avant la génération actuelle. L'ancêtre commun aux deux lignées remonte à quatre générations avant Yanbar et seulement une génération avant Dawazen. Avant ce point de bifurcation, les deux lignées sont constituées des mêmes noms, bien que leur succession soit loin d'être identique : quelques noms ont été soit ajoutés, soit omis, soit écrits de manière différente. Ces arbres généalogiques rattachent les hommes de la communauté actuelle des Berti au Prophète, 34 à 37 générations auparavant. Il est d'ailleurs intéressant de noter que bien que les membres des deux lignées descendent du même ancêtre, les Charafa conservent le titre de charîf (noble, descendant du Prophète) mais non la lignée Basanga. Conformément à l'accord susmentionné, le titre de charîf, qui est le plus prestigieux dans le domaine religieux, a été conservé uniquement par les Charafa. Quant aux Basanga, ils ont trouvé plus avantageux de mettre l'accent sur leur appartenance aux Berti autochtones plutôt que sur l'origine étrangère impliquée par le titre de charîf.

En évoquant l'adoption de l'islam et sa propagation parmi les Berti, il convient de mentionner le rôle que jouent les hommes de religion (fuqaha'; au sing. : faqîh). Le terme de faqîh, largement utilisé au nord du Soudan, a été traduit de plusieurs manières selon les intérêts ou l'optique des chercheurs. Ce terme a été ainsi traduit par " mendiant soufi » (Trimingham, 1949, 140), "saint homme " ou "chef spirituel», " médecin local », « cheikh religieux local », « pasteur local » (Trimingham, 1949, 140), etc. Bien que bon nombre des préceptes de l'islam aient été assimilés par les Berti et soient devenus partie intégrante de leur vie quotidienne, la nature de l'islam en tant que religion qui attribue une grande importance à la parole divine, c'est-à-dire au Coran, a permis aux fuqaha' lettrés de garder le monopole prestigieux de connaissances islamiques et linguistiques plus approfondies. Ainsi les rites islamiques des Berti ne peuvent-ils pas être pratiqués sans la présence d'un faqîh. Ce sont encore les fuqaha' qui officient lors des prières pratiquées à l'occasion des deuils, de la sécheresse ou autres catastrophes et qui président les prières collectives et celles des grandes fêtes religieuses. Il est rare de trouver un village berti qui n'ait pas de faqîh officiel chargé des rites pratiqués en public. Les fuqaha' s'occupent aussi des écoles coraniques où les Berti apprennent ne serait-ce que quelques versets du Coran. L'objectif ultime de ces institutions est bien sûr d'apprendre aux élèves à mémoriser le texte intégral du Coran, mais n'y parviennent exceptionnellement que quelques élèves qui deviennent euxmêmes, à leur tour, des fuqaha' professionnels. Durant ces derniers siècles, ce sont les 
fuqaha' qui ont largement contribué à la diffusion de l'islam au nord du Soudan. La diffusion de l'enseignement moderne dans cette région s'est d'ailleurs heurtée à la difficulté de rivaliser avec les fuqaha' qui y défendaient leur monopole éducatif dans cette région.

Nouvelles tendances

Depuis l'accession du Soudan à l'indépendance en 1956, plus de sept gouvernements se sont succédé à Khartoum, mais aucun d'entre eux n'a réellement essayé d'améliorer la vie des Soudanais en milieu rural. Néanmoins, et quelle que soit la distance qui les sépare du siège du gouvernement à Khartoum, les Berti ont été influencés par les politiques adoptées. Ceux qui étaient élèves d'écoles du secteur « moderne » et/ou ceux qui ont quitté leur village pour s'établir dans les grandes villes ont développé des affinités avec l'islam officiel. Certains Berti - ceux qui ont reçu une éducation plus approfondie que les autres ou qui ont préféré les grandes villes au milieu rural - ont été recrutés par des organisations islamiques extrémistes. Mais ces quelques éléments qui vivent presque exclusivement en milieu urbain n'ont que peu influencé la vie rurale en territoire berti. En 1983, la décision gouvernementale d'appliquer intégralement la loi islamique a suscité l'espoir d'un «Etat islamique idéal» et d'une véritable renaissance de l'islam. Mais le rêve n'a pas tardé pas à s'évanouir; amputations, pendaisons, flagellations et harcèlement ont vite terni l'image de la nouvelle loi. C'est d'ailleurs le mauvais accueil fait à cette législation au nord du Soudan (et surtout au Sud non musulman) qui a conduit à la chute du régime Nimeyri. Depuis, la plus grande confusion règne quand il s'agit de définir «l'islam authentique ».

Dans une communauté aussi marginale que celle des Berti, l'islamisation n'a pas entraîné de véritables changements dans la vie quotidienne, et les mouvements islamistes récents n'influencent guère la manière dont cette communauté conceptualise et/ou pratique l'islam. Les fondamentalistes musulmans qui, à l'occasion de leurs campagnes électorales, essaient de gagner des adeptes en son sein se trouvent obligés d'aborder des questions qui sont pour les Berti prioritaires par rapport aux questions religieuses dont les militants auraient préféré traiter avec eux. Comparée aux problèmes urgents que sont l'eau, les médicaments et l'alimentation; la chari'a, pour les Berti, est loin de représenter une priorité.

Conclusion

L'islam, religion universelle, n'a pas engendré une communauté musulmane homogène à travers le monde. Les diverses sociétés que nous qualifions de musulmanes ne présentent en effet que quelques traits communs (Geertz, 1968). Cette disparité du degré d'islamisation est beaucoup plus évidente en Afrique Noire musulmane que dans d'autres régions du monde islamique. Dans les sociétés africaines, l'islam est une fusion d'éléments de la foi musulmane et de pratiques appartenant au "paganisme» préislamique local. L'islam et les croyances païennes sont les deux éléments qui servent de base à un nombre infini de combinaisons structurant les pratiques sociales. Ces sociétés attribuent d'ailleurs plus d'importance à l'un ou l'autre de ces éléments de leur vie spirituelle selon les circonstances. Toutefois, malgré les croyances païennes fortement ancrées dans ces sociétés, nous sommes tentés de considérer ces dernières comme des sociétés musulmanes dans là mesure où elles se considèrent elles-mêmes et tiennent à être reconnues comme telles (voir Trimingham, 1949,47). Mais il ne faut pas oublier que dans toutes les sociétés musulmanes, il existe un très grand décalage entre l'islam en tant qu'idéologie et l'islam en tant que religion pratiquée par les musulmans. 
Le premier demeure un critère universellement reconnu, mais qui n'existe qu'en théorie. Essayer d'étudier diverses sociétés musulmanes à l'aune de ce critère universel qu'est l'islam canonique ne constitue donc qu'un exercice d'école.

Nous avons ainsi avancé que l'adhésion des Berti à l'islam s'est faite sur une base sélective. Souvent aussi, les Berti ont réinterprété certains enseignements de l'islam selon leur propre optique. Cela leur a permis de conserver des principes non islamiques propres à leur culture initiale. Bien que l'islam ait été introduit relativement tard dans leur culture, il y est bien enraciné. Cela est lié, dans une certaine mesure, au fait qu'ils se considèrent comme relevant d'une ascendance arabe. Pour eux, l'islam est moins une religion nouvelle à laquelle ils ont décidé d'adhérer parce qu'elle le méritait intrinsèquement, que la religion que professaient leurs ancêtres.

\section{BIBLIOGRAPHIE}

Gilsenan M., Recognizing Islam, Tauris, London, 1982.

Holy L., Neighbours and Kinsmen, A Study of the Berti Peopfe of Darfur, C. Hurst and Company, London, 1974.

Trimingham J. S.. Islam in the Sudan, Oxford University, 1949.

O’ Fahey R. S., State and Society in Dar Fur, St Martins Press, New-York, 1980.

Geertz C., Islam Observed: Religious Development in Marocco and Indonesia, New-Haven and London, 1968.

Lewis I. M., A Pastoral Democracy, London, 1966.

\section{NOTES}

1. A l'exception de quelques ajouts, les informations contenues dans cette étude sont exposées d'une manière plus détaillée dans ma thèse : Les hommes de religion et

l'alphabétisation dans la société berti. Thèse (inédite) de doctorat en Anthropologie sociale, Université de St. Andrews, 1983.

INDEX

Mots-clés : Soudan 
AUTEUR

ABDULAHI OSMAN AL-TOM

Université de Gezira 